\title{
Avaliação da independência funcional, qualidade de vida e frequência dos sintomas de depressão em mulheres sobreviventes ao câncer de mama
}

\author{
Evaluation of functional independence, quality of life and frequency of symptoms of \\ depression in women surviving breast cancer \\ Evaluación de independencia funcional, calidad de vida y frecuencia de síntomas de \\ depresión en mujeres sobrevivientes de cáncer de seno
}

Amanda Novaes Vieira Tertuliano ${ }^{1}$, Renata Alvarenga Vieira ${ }^{1}$, Leidiléia Mesquita Ferraz ${ }^{2 *}$, Jaqueline Ferreira Ventura Bittencourt ${ }^{1}$, Fabiane Rossi dos Santos Grincenkov ${ }^{1}$, Simone Meira Carvalho ${ }^{1}$.

\section{RESUMO}

Objetivo: Avaliar a independência funcional, qualidade de vida e frequência de sintomas depressivos em mulheres sobreviventes ao câncer de mama e verificar a presença de associação entre incapacidade funcional, qualidade de vida e a presença de sintomas depressivos. Métodos: Trata-se de um estudo observacional, transversal, com uma amostra de conveniência composta por 23 mulheres submetidas à mastectomia ou quadrantectomia com ou sem abordagem axilar. Os instrumentos utilizados foram questionário sociodemográfico, DASH, WHOQOL-bref, e HADS, que avaliavam, respectivamente, a funcionalidade do membro superior, qualidade de vida, e sintomas depressivos. Resultados: A função do membro superior apresentou um percentual $82,60 \%$ de incapacidade entre as participantes do estudo e foi associada a domínios físicos e ambientais de qualidade de vida. A qualidade de vida apresentou declínio no domínio físico e o domínio psicológico foi o melhor avaliado. Os sintomas depressivos foram observados em 47,83\% das participantes. Conclusão: A incapacidade funcional e os sintomas depressivos foram muito frequentes em mulheres sobreviventes ao câncer de mama. A incapacidade funcional foi associada aos domínios da qualidade de vida, mas não se associou a presença de sintomas depressivos. Destaca-se a importância da detecção e intervenção precoce dos comprometimentos que os tratamentos do câncer de mama podem desencadear.

Palavras-chave: Câncer de mama, Funcionalidade, Qualidade de vida, Depressão

\begin{abstract}
Objective: To assess functional independence, quality of life and frequency of depressive symptoms in women surviving breast cancer. Methods: This is an observational, cross-sectional study with a quantitative approach. 23 women who underwent mastectomy or quadrantectomy with or without axillary approach participated. The instruments used were a sociodemographic questionnaire, the WHOQOL-bref, DASH and HADS, which assessed, respectively, quality of life, upper limb functionality and depressive symptoms. Results: Selfassessment and general health perception had a positive evaluation, with the physical domain being the most impaired and the psychological domain being the best evaluated by WHOQOL-bref. The function of the upper limb showed a high percentage of disability. As for the depressive symptoms, $47.83 \%$ of the participants presented and $52.17 \%$ did not present these symptoms. Conclusion: Functional disability and depressive symptoms were very common in women who survived breast cancer, associated with poorer quality of life. The importance of early detection of the impairments that treatments can trigger is highlighted, in order to prevent or treat them, increasing the quality of life of these women.
\end{abstract}

Keywords: Breast cancer, Functionality, Quality of life, Depression.

${ }^{1}$ Universidade Federal de Juiz de Fora (UFJF), Juiz de Fora - MG

${ }^{2}$ Centro Universitário Estácio de Sá, Juiz de Fora - MG. *E-mail: enfleidi@gmail.com

SUBMETIDO EM: 8/2020

ACEITO EM: 9/2020

PUBLICADO EM: 12/2020 


\section{RESUMEN}

Objetivo: Evaluar la independencia funcional, la calidad de vida y la frecuencia de los síntomas depresivos en mujeres sobrevivientes de cáncer de mama. Métodos: Este es un estudio observacional de corte transversal con un enfoque cuantitativo. Participaron 23 mujeres que se sometieron a mastectomía 0 cuadrantectomía con 0 sin abordaje axilar. Los instrumentos utilizados fueron un cuestionario sociodemográfico, WHOQOL-bref, DASH y HADS, que evaluaron, respectivamente, la calidad de vida, la funcionalidad de las extremidades superiores y los síntomas depresivos. Resultados: La autoevaluación y la percepción general de la salud tuvieron una evaluación positiva, siendo el dominio físico el más afectado y el dominio psicológico el mejor evaluado por WHOQOL-bref. La función de la extremidad superior mostró un alto porcentaje de discapacidad. En cuanto a los síntomas depresivos, el 47,83\% de los participantes presentaron y el $52,17 \%$ no presentaron estos síntomas. Conclusión: La discapacidad funcional y los síntomas depresivos fueron muy comunes en las mujeres que sobrevivieron al cáncer de mama, asociadas con una peor calidad de vida. Se destaca la importancia de la detección temprana de las deficiencias que los tratamientos pueden desencadenar, para prevenirlas o tratarlas, aumentando la calidad de vida de estas mujeres.

Palabras clave: Cáncer de mama, Funcionalidad, Calidad de vida, Depresión.

\section{INTRODUÇÃO}

O câncer de mama é uma condição de saúde com elevados indicadores de morbimortalidade e desfechos adversos em saúde pública. Caracteriza-se como o segundo tipo mais comum de câncer no mundo e o primeiro entre as mulheres, respondendo por cerca de $25 \%$ dos casos novos a cada ano. No Brasil, para o triênio 2020-2022 estima-se a ocorrência de 66.000 novos casos de mama por ano. A região Sudeste é a segunda do país onde o câncer de mama feminino é o tipo mais frequente, sendo a taxa de incidência de 57,41 casos para cada 100 mil mulheres (BRASIL, 2019).

Nos estádios iniciais da doença, o tratamento é usualmente constituído por cirurgia e abordagem axilar (retirada de gânglios linfáticos) acompanhada de radioterapia, para destruir células remanescentes. Frequentemente, a abordagem axilar leva a comprometimentos funcionais do membro superior homolateral ao procedimento. Nos estádios mais avançados, além destas terapêuticas, é realizado o tratamento sistêmico com quimioterapia e hormonioterapia, visando controlar a doença (RECCHIA TL, et al., 2017).

Existem diversas técnicas cirúrgicas, conservadoras ou não. Dentre as não conservadoras, temos a mastectomia, que é a retirada da mama, com ou sem remoção do músculo peitoral. Dentre as conservadoras temos a quadrantectomia, que consiste na retirada de um quadrante mamário e da fáscia do músculo peitoral maior, e setorectomia, que é a retirada de um setor mamário (COSTA ID, et al., 2018; MENDES IS, et al., 2014).

As complicações do tratamento do câncer de mama são extensas, destacando-se aquelas decorrentes da intervenção cirúrgica, como: dor, retrações cicatriciais, lesão nervosa, seroma, disfunções respiratórias, alteração da amplitude de movimento (ADM) do ombro, diminuição de força muscular e linfedema (RECCHIA TL, et al., 2017).

As alterações linfáticas podem surgir em qualquer período após a cirurgia como complicações importantes, de elevada prevalência e com efeitos que abalam a qualidade de vida (CESAR ESL, et al., 2017; MACHADO MX, et al., 2017; RECCHIA TL, et al., 2017).

O tratamento do câncer mamário associa-se a desfechos psicológicos, dentre os quais a depressão é uma das condições mais frequentes, sendo influenciada pelo tempo decorrido da cirurgia e os tipos de tratamentos realizados, em especial a quimioterapia (JURADO SR, et al., 2019; FERREIRA BPB, et al., 2016; SANTOS MS, et al., 2019). 
As alterações provocadas pelos tratamentos também geram um déficit no desempenho ocupacional que resultam, muitas vezes, no abandono de algumas atividades diárias nas dimensões do autocuidado, de atividades domésticas, de lazer, de trabalho e de participação social, afetando a qualidade de vida e desencadeando um sentimento de incapacidade (COSTA ID, et al., 2018; FERREIRA AS, et al., 2015; SANTOS MS, et al., 2019). Neste contexto, a abordagem terapêutica é imprescindível para manutenção da funcionalidade, visando a independência das mulheres no desempenho de suas atividades pessoais, profissionais e de lazer (MACHADO MX, et al., 2017; RECCHIA TL, et al., 2017). Compreende-se que um olhar integral se torna importante para recuperação da saúde e um melhor processo de reabilitação (CARVALHO SM, et al., 2018; DIAS LV, et al., 2017; SILVA MB, et al., 2016).

Neste cenário, destaca-se a importância de compreender e identificar os impactos que os tratamentos do câncer de mama podem trazer na vida das mulheres. Assim como contribuir para a promoção de abordagens terapêuticas que visem a independência funcional. Portanto, o estudo se propôs a avaliar a funcionalidade, a qualidade de vida e a depressão, objetivando identificar a frequência destas condições de saúde nas participantes do estudo e também verificar a associação entre a incapacidade funcional em membro superior, presença de sintomas depressivos e a qualidade de vida em mulheres submetidas ao tratamento de câncer mamário.

\section{MÉTODOS}

Trata-se de um estudo quantitativo observacional do tipo transversal. A amostra do estudo foi de conveniência, constituída por mulheres sobreviventes ao câncer de mama, atendidas em um ambulatório de um hospital universitário do estado de Minas Gerais, no período de março de 2017 a abril de 2018. Foram incluídas no estudo mulheres submetidas à cirurgia para o tratamento do câncer de mama (mastectomia ou quadrantectomia), com ou sem reconstrução mamária, e idade mínima de 18 anos. Foram excluídas aquelas que apresentaram evolução da doença; déficit cognitivo e/ou relato de alteração funcional em membro superior antes do diagnóstico do câncer.

Participaram um total de 23 mulheres, que foram avaliadas por meio de um questionário com dados sociodemográficos e clínicos e, a seguir, foram aplicados os instrumentos para a avaliação das variáveis da pesquisa. Este estudo foi aprovado pelo Comitê de Ética da Universidade Federal de Juiz de Fora (UFJF), parecer 1.047.539, de 4 de maio de 2015. As mulheres que aceitaram participar foram informadas sobre os objetivos, os instrumentos a serem utilizados e as implicações éticas da pesquisa, por meio do Termo de Consentimento Livre e Esclarecido.

A variável dependente do estudo foi a funcionalidade, avaliada pela presença ou ausência de incapacidade funcional do membro superior homolateral ao procedimento cirúrgico, operacionalizada pela aplicação do instrumento Disabilities of Arm Shoulder and Hand (DASH). Este instrumento foi validado para o português, em 2003 por Orfale, com objetivo de avaliar o desempenho funcional e sintomas físicos, sendo dois itens relacionados à função física, seis relacionados aos sintomas e três relativos às funções sociais.

O escore varia de 0 a 100, cujas respostas tem escore variando entre 1 a 5 , sendo pior a funcionalidade quanto mais próximo de 100 (RECCHIA TL, et al., 2017; ORFALE AG, et al., 2005). Escores abaixo ou igual a 25 pontos, indicam pouca ou nenhuma incapacidade. No indicativo de algum nível de incapacidade, as pontuações estão entre os dois quartis (25 e 75). Os escores com quartil mais alto, caracteriza extrema incapacidade funcional (RECCHIA TL, et al., 2017).

Como variáveis independentes, foram avaliadas a qualidade de vida (QV) e os sintomas depressivos. A QV foi operacionalizada pelo instrumento World Health Organization Quality of Life - bref (WHOQOL-bref). Este possui 26 questões, sendo duas relacionadas à QV geral, com um item correspondente a este aspecto e o outro à percepção da saúde, e as 24 outras questões são divididas em domínios: físico, psicológico, relações sociais e meio ambiente. As respostas têm pontuação na escala Likert, variando de 1 a 5 pontos, onde escores mais altos predizem melhor QV. Esse questionário não possui um ponto de corte e nem um escore único, então as análises dos resultados foram realizadas de acordo com cada domínio. 
Os sintomas depressivos foram avaliados por meio da Escala Hospitalar de Ansiedade e Depressão (HADS), desenvolvida por Zigmond e Snaith, em 1983, para avaliar sintomas de ansiedade e depressão. Esta foi validada no Brasil, e é amplamente utilizada em estudos brasileiros para rastrear sintomas depressivos e de ansiedade em pacientes com câncer (FERREIRA BPB, et al., 2016). É constituída por 14 questões, sendo 7 referentes à ansiedade e 7 à depressão. Para cada pergunta, atribui-se nota de 0 a 3 , podendo pontuar de 0 a 21 em cada subescala, sendo que pontuação igual ou maior que oito é sugestiva de transtornos.

$\mathrm{Na}$ análise estatística, foi aplicado o teste Qui-Quadrado de Pearson para verificar a associação entre a variável dependente, presença ou ausência de incapacidade, e cada variável independente do estudo, sendo elas a QV e sintomatologia depressiva, por meio do programa estatístico SPSS ${ }^{\circledR}$ (versão 17). O teste t de Student foi utilizado para avaliar as diferenças entre as médias entre dois grupos (pouca ou nenhuma incapacidade e algum nível de incapacidade) formados a partir dos escores do DASH. Para a análise de associação foi utilizado o teste de Qui-Quadrado de Pearson para avaliar os dados categóricos.

\section{RESULTADOS E DISCUSSÃO}

A amostra apresentou uma média etária de 53,39 anos, sendo semelhante à de mulheres com histórico de câncer de mama dos estudos de Barbosa PA, et al. (2017) e Sousa ALV, et al. (2014). Estes estudos também corroboram os dados do INCA, segundo os quais, câncer de mama em mulheres antes dos 35 anos são mais raros e acima desta idade a incidência cresce progressivamente, principalmente após os 50 anos (BRASIL, 2019). A idade pode interferir também na QV destas mulheres, sendo que as mais novas tendem a apresentar uma pior aceitação da situação delicada de saúde (SOUSA ALV, et al., 2014).

Quanto às características clínicas, pouco mais da metade das mulheres foram submetidas à mastectomia com abordagem axilar $(52,16 \%)$, seguida de mastectomia sem abordagem axilar $(26,10 \%)$ e quadrantectomia com abordagem axilar (21,74\%), sendo que $52,17 \%$ realizaram reconstrução mamária. O tempo médio decorrido da cirurgia até a entrevista foi 5,04 anos ( $D P \pm 3,17)$.

Quanto ao tratamento, 60,87\% realizaram quimioterapia, 52,17\% radioterapia, e 86,96\% hormonioterapia. Dentre as complicações relatadas, $17,40 \%$ das mulheres sofreram radiodermite, $52,17 \%$ linfedema, $69,57 \%$ dor, 91,30\% apresentou limitação da ADM de membros superiores (MMSS) e 86,96\% alteração de sensibilidade ao redor da cirurgia e/ou em MMSS.

Nos procedimentos com dissecção axilar, a despeito do tipo de cirurgia, os riscos de complicações podem ser maiores e afetam negativamente a QV. As complicações aumentam à medida que as técnicas cirúrgicas se tornam mais agressivas, maior número de linfonodos são dissecados e, contribuindo para um maior comprometimento físico (BARBOSA PA, et al., 2017; RECCHIA TL, et al., 2017).

O tempo médio de cirurgia desta amostra foi de aproximadamente cinco anos. No estudo de Recchia TL, et al. (2017), após cinco anos decorridos da cirurgia, houve impacto regular quanto à funcionalidade do membro homolateral à cirurgia, com consequente diminuição da $\mathrm{QV}$, reforçando os achados do presente estudo. Correlacionando o escore do DASH e o tempo decorrido da cirurgia, observou-se que, quanto maior o tempo, maiores são as complicações que interferem na funcionalidade do membro superior. Costa ID, et al. (2018) defendem que, através do perfil clínico e funcional das mulheres, é possível estabelecer um tratamento pautado no modelo biopsicossocial, podendo melhorar a adesão ao tratamento.

Em relação à $Q V$, foi identificada pior escore no domínio físico $(11,75 \pm 3,19)$ e o maior escore no domínio psicológico $(14,29 \pm 2,46)$. A autoavaliação da QV foi considerada como positiva para as participantes do estudo $(13,13 \pm 2,94)$ (Tabela 1). Tal resultado pode ser justificado pelo fato de estarem inseridas no ambulatório de fisioterapia e possuírem mais oportunidades de orientações profissionais, de cuidados e de convívio social, o que favorece uma melhora global em seu estado de saúde (CARVALHO SM, et al., 2018; NETO EAA, et al., 2017).

A avaliação da QV dada pelo WHOQOL-bref mostra que o domínio físico foi o menor escore, assemelhando-se aos resultados de outros autores que aplicaram o mesmo instrumento (NETO EAA, et al., 2017; SOUSA ALV, et al., 2014). 
Tabela 1 - Qualidade de vida (QV) em mulheres com histórico de câncer de mama usuárias do ambulatório de fisioterapia - $2018(\mathrm{~N}=23)$.

\begin{tabular}{ccccccc}
$\begin{array}{c}\text { Domínios de } \\
\text { Qualidade de Vida }\end{array}$ & Média & $\begin{array}{c}\text { Desvio } \\
\text { Padrão }\end{array}$ & $\begin{array}{c}\text { Coeficiente } \\
\text { de Variação }\end{array}$ & $\begin{array}{c}\text { Valor } \\
\text { Mínimo }\end{array}$ & $\begin{array}{c}\text { Valor } \\
\text { Máximo }\end{array}$ & Amplitude \\
\hline $\begin{array}{c}\text { Físico } \\
\text { Psicológico }\end{array}$ & 11,75 & 3,19 & 27,15 & 6,86 & 18,29 & 11,43 \\
Relações Sociais & 14,29 & 2,46 & 17,22 & 10,00 & 18,00 & 8,00 \\
Meio Ambiente & 13,39 & 2,53 & 18,92 & 8,00 & 18,00 & 10,00 \\
Autoavaliação da QV & 12,70 & 2,43 & 19,17 & 8,00 & 16,00 & 8,00 \\
\hline Total & 13,13 & 2,94 & 22,42 & 6,00 & 18,00 & 12,00 \\
\hline
\end{tabular}

Legenda: QV = WHOQOL-bref - World Health Organization Quality of Life. Fonte: Tertuliano ANV, et al., 2020.

$\mathrm{Na}$ avaliação da independência funcional, foi observada maior frequência de mulheres com incapacidade funcional em membro superior, onde resultados entre 25 e 75 pontos indicavam algum nível de incapacidade $(82,60 \%)$ e pontuações abaixo ou igual a 25 pontos indicavam pouca ou nenhuma incapacidade $(17,40 \%)$ (Tabela 2).

A maioria das participantes do estudo foi submetida à mastectomia com abordagem axilar. Das que realizaram só mastectomia, metade apresentou algum nível de incapacidade e a outra metade apresentou pouca ou nenhuma incapacidade. Isso pode se justificar por diversos fatores que independem do tipo de cirurgia como, por exemplo, o tempo decorrido, restrição de movimento por dor e medo de realizar a cinesioterapia, falta de informações no pré e pós-operatório e falta de encaminhamento para a fisioterapia precocemente (NETO EAA, et al., 2017; COSTA ID, et al., 2018).

Tabela 2 - Função do membro superior de mulheres com histórico de câncer de mama usuárias do ambulatório de fisioterapia - $2018(\mathrm{~N}=23)$.

\begin{tabular}{ccc}
\hline DASH (Escala de $\mathbf{0}$ a 100) & N & $\%$ \\
\hline Geral & 23 & 100 \\
Algum nível de incapacidade & 19 & 82,60 \\
Pouca ou nenhuma incapacidade & 4 & 17,40 \\
\hline
\end{tabular}

Legenda: DASH = Disabilities of Arm Shoulder and Hand. Fonte: Tertuliano ANV, et al., 2020.

No presente trabalho, todas as mulheres que foram submetidas à quadrantectomia com abordagem axilar tiveram algum nível de incapacidade de acordo com os resultados do DASH. Nava LP, et al. (2016) expõe que complicações cirúrgicas tais como o linfedema, a dor, a restrição de movimento do braço, bem como os quadros de depressão e ansiedade interferem na QV e no processo de recuperação.

Para Mendes IS, et al. (2014), a cirurgia de retirada da mama pode ocasionar dor, em especial nas mulheres mais idosas, podendo estar associada a comorbidades ligadas ao envelhecimento. Ademais, a cirurgia gera prejuízos em longo prazo, originando déficits no desempenho das tarefas diárias, prejudicando o estado geral de saúde e a capacidade física e social. Há que se considerar que a dor induz a disfunções físicas que impactam no equilíbrio psicológico e na QV.

Carvalho FN, et al. (2014) demostraram que o encaminhamento tardio pode diminuir as possibilidades de uma melhor reabilitação física funcional. Segundo Mendes IS, et al. (2014), a realização de um programa de atividade física específica após a cirurgia possibilita, às mulheres sobreviventes ao câncer, um incremento na movimentação do braço e maior sensação de bem-estar, promovendo um retorno precoce às atividades cotidianas. 
Pouco mais da metade $(52,17 \%)$ das participantes da pesquisa não apresentaram sintomas depressivos, avaliados através da escala HADS (Tabela 3). Ferreira AS, et al. (2015), estudando mulheres com câncer mamário em nível ambulatorial, encontraram uma incidência de depressão de $26,8 \%$ e de $24,74 \%$ de ansiedade.

Jurado SR, et al. (2019) reportam que altos níveis de ansiedade e depressão são frequentes no período do diagnóstico e início dos tratamentos, pelo temor da morte e agressividade das terapêuticas, considerando uma tendência de queda desses níveis em longo prazo.

Apenas quatro mulheres apresentaram complicações com a radioterapia, mas vale ressaltar que este tratamento também está relacionado às morbidades do membro superior, causando efeitos como alteração da sensibilidade, dor e fibrose (RECCHIA TL, et al., 2017).

Nesta amostra, 95,65\% relataram ter tido complicações pós-cirúrgicas, com maior prevalência da limitação da ADM, seguida de alteração de sensibilidade, dor e linfedema, corroborando os estudos de Sousa ALV, et al. (2014).

Tabela 3 - Sintomas depressivos em mulheres com histórico de câncer de mama usuárias do ambulatório de fisioterapia $-2018(\mathrm{~N}=23)$.

\begin{tabular}{ccc}
\hline HADS - Sintomas depressivos & $\mathbf{N}$ & $\%$ \\
\hline Geral & 23 & 100 \\
Sem sintomas depressivos (0-8) & 12 & 52,17 \\
Com sintomas depressivos (>=9) & 11 & 47,83 \\
\hline
\end{tabular}

Legenda: HADS = Escala Hospitalar de Ansiedade e Depressão. Fonte: Tertuliano ANV, et al., 2020.

A variável Incapacidade funcional e QV (por domínios) apresentaram associações comparando as mulheres que possuem pouca ou nenhuma incapacidade com aquelas que possuem algum nível de incapacidade (Tabela 4).

A associação entre estas variáveis foi verificada para os domínios físico e meio ambiente, que constam no instrumento WHOQOL-bref. O domínio físico compreende aspectos: relacionados à dor e ao desconforto, relativos à energia e à fadiga, pertinentes ao sono e ao repouso, dentre outros.

Já o domínio meio ambiente aborda diversas facetas: associadas à segurança física e à proteção, relacionadas ao ambiente no lar, tocante aos recursos financeiros, e aos cuidados de saúde e sociais, por exemplo).

O grupo de mulheres que possuíam pouca ou nenhuma incapacidade apresentaram melhor QV referenciada pelos domínios físico e meio ambiente quando comparadas às pacientes que possuíam algum nível de incapacidade. Na avaliação da QV nos domínios psicológico, relações sociais, QV global e percepção de saúde, não houve diferença significativa para as mulheres com pouca ou nenhuma incapacidade e aquelas com algum nível de incapacidade quanto à QV nesta amostra.

Tabela 4 - Associação entre Incapacidade Funcional em membro superior e QV em mulheres sobreviventes ao câncer de mama usuárias do ambulatório de fisioterapia - 2018 ( $\mathrm{N}=23)$.

\begin{tabular}{cccccc}
\hline DASH & Físico & Psicológico & $\begin{array}{c}\text { Relações } \\
\text { sociais }\end{array}$ & $\begin{array}{c}\text { Meio } \\
\text { Ambiente }\end{array}$ & $\begin{array}{c}\text { QV global e percepção } \\
\text { saúde }\end{array}$ \\
\hline PNI & $75,89 \%$ & $66,66 \%$ & $58,33 \%$ & $64 \%$ & $57 \%$ \\
ANI & $43,10 \%$ & $65,62 \%$ & $59,37 \%$ & $53,91 \%$ & $55 \%$ \\
Valor $p$ & $\mathrm{p}<0,001^{*}$ & $\mathrm{p}=0,75$ & $\mathrm{p}=0,96$ & $\mathrm{p}=0,01^{*}$ & $\mathrm{p}=0,08$ \\
IC 95\% & $-8,17$ a $-2,46$ & $-3,33$ a 2,42 & $-2,9$ a 3,04 & $-3,15 \mathrm{a}-0,62$ & $-6,06$ a 0,32 \\
\hline
\end{tabular}

Legenda: $\mathrm{QV}=$ Qualidade de Vida; WHOQOL-bref=World Health Organization Qualityof Life; DASH= Disabilities of Arm Shoulder and Hand; $\mathrm{PNI}=$ pouca ou nenhuma incapacidade; $\mathrm{ANI}=$ algum nível de incapacidade; *significativo para $\mathrm{p} \leq 0,05$; IC = Intervalo de Confiança.

Fonte: Tertuliano ANV, et al., 2020. 
Os resultados da avaliação da presença de associação entre a incapacidade funcional avaliada pelo DASH e a QV avaliada pelo WHOQOL-bref mostrou que houve associação com o domínio físico, sendo que, as mulheres que tiveram algum nível de incapacidade deste estudo, foram as que apresentaram baixa pontuação neste domínio. Isso indica que o grupo das mulheres que tiveram algum nível de incapacidade, também apresentou impacto negativo semelhante na QV, relacionada às condições físicas (FERNANDES MR, 2015).

Jurado SR, et al. (2019) destacam que a fadiga, a dor e o comprometimento do sono são fatores que podem perdurar por anos após o término da quimioterapia, e implicam na funcionalidade, na independência para realização de suas tarefas diárias, gerando sintomas depressivos. Cunha NF (2018) menciona que a fadiga secundária à quimioterapia gera desânimo e limitações na realização das atividades cotidianas, desencadeando alterações de humor, angústia e impaciência. Cesar ESL, et al. (2017) referem que a ausência de sintomas dos efeitos colaterais da quimioterapia está diretamente ligada a uma percepção de melhor QV.

Houve associação entre o domínio meio ambiente com a incapacidade funcional, conforme também destacado no estudo de Recchia TL, et al (2017). O segundo domínio mais afetado na avaliação de QV no presente estudo foi o ambiental, que vai de encontro com o estudo de Sousa ALV, et al. (2014), apontando que as mulheres não se sentem totalmente seguras no meio onde vivem. O domínio pontuado mais alto na avaliação de QV foi o psicológico, semelhante ao estudo supracitado, que apesar de ter tido um bom resultado, é um domínio que influencia muito na vida destas mulheres.

Não houve associação significativa entre incapacidade funcional com os demais domínios de QV, e isso se justifica pelo fato do DASH só possuir correlação com o domínio físico do WHOQOL-bref, uma vez que não se correlaciona fortemente com instrumentos que avaliam diferentes conceitos, que não sejam relacionados com os aspectos físicos do paciente, já que ele ressalta principalmente a função física como forma de avaliar as desordens musculoesqueléticas (RECCHIA TL, et al., 2017; FERNANDES MR, 2015).

Em um estudo realizado com mulheres submetidas a tratamento cirúrgico para o câncer de mama, 78\% foram submetidas à mastectomia e $72 \%$ foram submetidas à dissecção axilar, com tempo decorrido de cirurgia de 1 ano e meio, aproximadamente, tiveram resultados positivos na avaliação da QV e baixa incapacidade funcional (CARVALHO FN, et al., 2014). Esses resultados podem ser justificados pelo fato de o estudo ter ocorrido em um hospital de referência em oncologia, pela abordagem pré-cirúrgica, tendo como conduta orientar sobre os cuidados necessários, identificar fatores que podem diminuir a funcionalidade e oferece tratamento fisioterapêutico precoce (NAVA LP, et al., 2016; CARVALHO FN, et al., 2014).

Quanto à incapacidade funcional e à sintomatologia depressiva em mulheres com histórico de câncer de mama, não foi identificada neste estudo associação estatística, dado o $p$-valor $=0,924$ considerando o nível de significância de 5\% para esta amostra (Tabela 5). Acredita-se que isto ocorreu pela mesma justificativa atribuída à não correlação do DASH com instrumentos que avaliam aspectos diferentes que não os físicos (FERNANDES MR, 2015). Não foram encontrados pelos pesquisadores estudos que abordassem a avaliação de sintomas depressivos fazendo relações com a funcionalidade.

Tabela 5 - Associação entre Incapacidade Funcional em membro superior e sintomas depressivos em mulheres sobreviventes ao câncer de mama usuárias do ambulatório de fisioterapia - 2018.

\begin{tabular}{cccc}
\hline \multirow{2}{*}{ Incapacidade funcional (DASH) } & \multicolumn{2}{c}{ Sintomas depressivos (HAD) } & \multirow{2}{*}{ Total } \\
\cline { 2 - 4 } & Não & Sim & \\
\hline PNI & 2 & 2 & 4 \\
\hline ANI & 10 & 9 & 19 \\
\hline Total & 12 & 11 & 23 \\
\hline
\end{tabular}

Legenda: Qui-quadrado de Pearson: $\mathrm{p}$-valor $=0,924 ;{ }^{*} \mathrm{PNI}=$ pouca ou nenhuma incapacidade; *ANI = algum nível de incapacidade; *Sim = com sintomas depressivos; *Não = sem sintomas depressivos.

Fonte: Tertuliano ANV, et al., 2020.

No presente estudo, do grupo de mulheres que tinham pouca ou nenhuma incapacidade, metade apresentaram depressão e a outra não apresentaram. O grupo daquelas que tinham algum nível de 
incapacidade, também apresentaram resultados semelhantes quanto à frequência de sintomatologia depressiva.

Alguns estudos apontam que a depressão poderia estar mais relacionada a fatores pessoais como, ser mais jovem, apresentar comprometimento psicológico anterior, do que especificamente com o tratamento ou a doença propriamente dita (JURADO SR, et al., 2019; FERREIRA AS, et al., 2015;).

Costa ID, et al. (2018) e Ferreira AS, et al. (2015) apontam que mulheres com menor escolaridade e que receberam tratamentos adjuvantes, possuem mais riscos de permanecer em quadros de ansiedade e depressão por um longo período.

Costa ID, et al. (2018) e Cunha NF (2018) destacam que as complicações decorrentes das terapêuticas para o câncer limitam a realização de atividades do dia-a-dia, impactando negativamente na funcionalidade e na QV das mulheres.

Jurado SR, et al. (2019) e Ferreira BPB, et al. (2016), destacam que as intervenções realizadas de forma individual, como relaxamento, exercícios fisioterapêuticos, ou atividades em grupo, ajudaram a reduzir os sintomas depressivos.

Nava LP, et al. (2016) ressaltam que os tratamentos que visam restabelecer os movimentos, a funcionalidade e o retorno das mulheres às atividades cotidianas, estimulam sentimentos de independência e uma melhor percepção da QV, o que impacta positivamente sobre a saúde.

Segundo Dias LV, et al. (2017), a prática de exercícios melhora a força muscular e resistência, flexibilidade e aptidão cardiorrespiratória. Entretanto, as sequelas físicas como dor, alteração de sensibilidade e perda de força muscular do braço podem prejudicar a realização das atividades diárias.

Neste sentido, Cunha NF (2018), Dias LV, et al. (2017) e Silva MB, et al. (2016), entendem que os profissionais de saúde devem se preocupar com a funcionalidade das mulheres acometidas pelo câncer mamário tanto no pós-operatório imediato como no tardio, já que implica em benefícios para o enfrentamento da doença e seus tratamentos e favorece a prevenção de sofrimento psicológico e os cuidados com a saúde.

Esta pesquisa apresentou limitações tais como pequeno tamanho amostral devido ao tipo de amostra por conveniência. Foi uma amostra heterogênea no que diz respeito às características clínicas e idade, podendo ter gerado viés. O uso do WHOQOL-bref também dificultou de certa forma, a obtenção de um resultado preciso, uma vez que não possui um escore geral, mas sim avalia a QV em domínios.

\section{CONCLUSÃO}

A incapacidade funcional e os sintomas depressivos foram muito frequentes em mulheres sobreviventes ao câncer de mama. A incapacidade funcional em membro superior esteve associada a pior qualidade de vida relacionada a fatores ambientais e a domínios físicos dessas mulheres. Assim, compreende-se que é de extrema importância a detecção precoce dos possíveis comprometimentos que o adoecimento por câncer e seus tratamentos podem desencadear. Para tanto, faz-se necessário realizar abordagens precoces por uma equipe composta por profissionais de diversas áreas, que possa acolher e tratar todos os aspectos que abrangem a saúde, visando a prevenção de quadros incapacitantes e sintomas depressivos para promover uma melhor qualidade de vida de mulheres com histórico de câncer de mama.

\section{REFERÊNCIAS}

1. BARBOSA PA, et al. Qualidade de vida em mulheres com câncer de mama pós-intervenção cirúrgica em uma cidade da zona da mata de Minas Gerais, Brasil. Rev. Bras. Saúde Matern. Infant., 2017; 17(2): 401-416.

2. BRASIL. 2019. In: Ministério da Saúde. Instituto Nacional de Câncer José Alencar Gomes da Silva (INCA). Estimativa 2020: Incidência de câncer no Brasil. Rio de Janeiro: Ministério da Saúde, INCA; 2019.

3. CARVALHO FN, et al. Functionality in women with breast cancer: the use of International Classification of Functioning, Disability and Health (ICF) in clinical practice. Journal of Physical Therapy Science, 2014; 26(5): 721-730. 
4. CARVALHO SM, et al. Corpo, funcionalidade, espiritualidade e câncer de mama. In: FILGUEIRAS, et al. (orgs). Câncer de mama: interlocuções e práticas interdisciplinares. Curitiba: Appris, 2018; 137-155.

5. CESAR ESL, et al. Qualidade de vida de mulheres com câncer mamário submetidas à quimioterapia. Revista Rene, 2017; 18(5): 679-686.

6. COSTA ID, et al. Utilização de um core set da cif para a descrição da atividade e participação de mulheres submetidas ao tratamento cirúrgico para o câncer de mama. Revista Interdisciplinar Ciências Médicas, 2018; 1(2): 4-14.

7. CUNHA NF. Experiências de mulheres com câncer de mama no manejo da fadiga secundária à quimioterapia. Dissertação (Mestrado em Ciências da Saúde) - Faculdade de Medicina. Universidade Federal de Uberlândia, Uberlândia, 2018; 90 p.

8. DIAS LV, et al. Mastectomized woman in breast cancer: experience of everyday activities. Rev Fun Care Online. 2017 out/dez; 9(4): 1074-1080.

9. FERNANDES MR. Correlation between functional disability and quality of life in patients with adhesive capsulitis. Acta Ortopédica Brasileira, 2015; 23(2): 81-84.

10. FERREIRA AS, et al. Câncer de mama: estimativa da prevalência de ansiedade e depressão em pacientes em tratamento ambulatorial. Arquivos de Ciências da Saúde da UNIPAR, 2015; 9(3): 185-189.

11. FERREIRA BPB, et al. Prevalência de ansiedade e depressão em pacientes oncológicos e identificação de variáveis predisponentes. Revista Brasileira de Cancerologia, 2016; 62(4): 321-328.

12. JURADO SR, et al. Sintomas depressivos em mulheres com câncer de mama submetidas à quimioterapia e radioterapia: uma revisão integrativa. Revista Nursing, 2019; 22(253): 2967-2972.

13. MACHADO MX, et al. Significados do câncer de mama para mulheres no contexto do tratamento quimioterápico. Physis Revista de Saúde Coletiva, 2017; 27(3): 433-451.

14. MENDES IS, et al. Correlação da dor e qualidade de vida de mulheres pós-tratamento cirúrgico de câncer de mama. Revista O Mundo da Saúde, 2014; 38(2): 189-196.

15. NAVA LP, et al. Funcionalidade de membro superior e qualidade de vida de mulheres com câncer de mama submetidas a tratamento fisioterapêutico. Rev. Aten. Saúde, 2016; 14(48): 21-26.

16. NETO EAA, et al. Quality of life post-mastetomywommen living in a semi-arid region of Brazil. International Journal of Envirnmental Reseratch and Public Healt, 2017; 14(601): 1-9.

17. ORFALE AG, et al. Translation into Brazilian Portuguese, cultural adaptation and evaluation of the reliability of the Disabilities of the Arm, Shoulder and Hand Questionnaire. Brazilian Journal of Medical and Biological Research, 2005; 38(2): 293-302.

18. RECCHIA TL, et al. Upper Limb Functionality and Quality of Life in Women with Five-Year Survival after Breast Cancer Surgery. Revista Brasileira de Ginecologia e Obstetrícia, 2017; 39(3): 115-122.

19. SANTOS MS, et al. Implicações da mastectomia na autoestima da mulher. Revista Eletrônica Acervo Saúde, 2019 , 29: e1124.

20. SILVA MB, et al. Trajetória de vida de mulheres mastectomizadas à luz do discurso do sujeito coletivo. Revista Cuidado é Fundamental, 2016; 8(23): 4365-4375.

21. SOUSA ALV, et al. Análise da qualidade de vida em mulheres mastectomizadas atendidas no ambulatório do HBDF. Comunicação em Ciências da Saúde, 2014; 25(1): 13-24. 